\title{
Liposuction-induced metabolic alterations - the effect on insulin sensitivity, adiponectin, leptin and resistin
}

\author{
Magdalena Gibas-Dorna', Piotr Turkowski², Małgorzata Bernatek³ , Kinga Mikrut', \\ Justyna Kupsz', Jacek Piąt ek \\ ${ }^{1}$ Department of Physiology, Poznan University of Medical Sciences, 6 Swiecicki Str., 60-781 Poznan, Poland \\ ${ }^{2}$ Samir Ibrahim Mandala Beauty Clinic, Chwiałkowskiego Str. 28/7, 61-553 Poznan, Poland \\ ${ }^{3}$ Chair of Social Medicine, Poznan University of Medical Sciences, 5 Rokietnicka Str., 60-806 Poznan, Poland
}

\begin{abstract}
Liposuction surgically removes subcutaneous abdominal tissue (SAT) and has almost no effect on visceral abdominal tissue (VAT) depot. However, some authors suggest that deep layers of SAT are functionally similar to VAT and the amount of deep subcutaneous abdominal adipose tissue is strongly related to insulin resistance in a manner nearly identical to that of visceral adiposity. Moreover, SAT determines leptin secretion which indirectly reflects the level of insulin sensitivity in the body. Thus, the immediate removal of SAT could potentially affect metabolic profile of a patient. The current data are conflicting and cannot bring a clear evidence suggesting that liposuction itself results in important metabolic outcomes and, on the other hand, cannot exclude such a possibility. This review summarizes the liposuction-induced metabolic changes with regard to release of major adipokines and insulin sensitivity.
\end{abstract}

Keywords: liposuction, insulin sensitivity, adiponectin, leptin, resistin, metabolic outcomes.

\section{Introduction}

According to International Society of Aesthetic Plastic Surgery liposuction was the second most frequently performed aesthetic operation in 2013. As with any surgery liposuction carries risks, however, in recent years, improved techniques have made liposuction safer, easier, and less painful. Thus, any intervention that immediately decreases adiposity and is relatively safe could be a viable method not only for aesthetic purpose but also for increasing the efficiency of insulin and improving metabolic profile, especially when combined with regular exercise and proper diet. Beneficial change in adipose tissue metabolism may be achieved directly or indirectly by modified release of metabolically active hormones/adipokines, modulated response to these hormones or by increased activity of sympathetic afferents to the fat cell. This paper presents the current state of knowledge on liposuction-induced metabolic changes with regard to release of major adipokines.

\section{Types, distribution and function of adipose tissue}

Adipose tissue is a metabolically active organ involved in multiple functions. It serves as a buffer against influx of dietary fat and energy depot, plays role in the processes of satiety control, thermoregulation, reproduction, and exhibits immunomodulatory and endocrine activities. As an endocrine organ adipose tissue secretes hormonally active factors including leptin, adiponectin, resistin, visfatin, interleukin 6 (IL6), tumor necrosis factor (TNF), and many others, and plays role in hormonal metabolism of sex hormones and glucocorticoids [1]. 
The division of adipose tissue into two main types, white and brown, reflects functional and anatomical differences. White adipose tissue (WAT) is localized under skin and is designed for energy storage whereas brown adipose tissue (BAT) is present around kidneys, adrenals, aorta, and within mediastinum and neck. BAT plays role in secretion of inflammatory mediators, dissipates energy and generates heat. Energetic utilization of fats is regulated by beta-3 adrenergic receptors activation and/or in the mechanism of postprandial thermogenesis, which is due to leptin activity and signals from ventromedial hypothalamus in response to postprandial levels of glucose and insulin. Depending on body's demands, white adipose tissue acts as a potent buffer maintaining constant level of fatty acids in the circulation by storing lipids in the form of triglycerides and esterified form of cholesterol, and also releasing free fatty acids. WAT is also the major site of leptin and adiponectin production. [2]. The most important localizations of WAT are visceral abdominal tissue (VAT), subcutaneous abdominal tissue (SAT) and femoral and gluteal subcutaneous regions. Different anatomic distribution of WAT is related with different function, metabolic and endocrine activity. 2/3 of WAT is present in SAT, while the rest is found around viscera, in the retroperitoneal and gonadal depots, in the mammary glands, liver and skeletal muscles.

Abdominal obesity, also known as a central obesity, results from accumulation of fat in both SAT and VAT and serves as a risk factor for insulin resistance, which in turn favors development of diabetes type II, dyslipidemia, arteriosclerosis and chronic inflammation with inflammatory cytokines production (eg. TNF alpha, IL6, IL1) and inhibition of anti-inflammatory cytokines release (eg. adiponectin, IL10) [3]. The expression and secretion of adipokines in adipose tissue vary according to the adipocyte size and number and to the adipose tissue depot. For example, adiponectin and leptin expression and secretion are higher in SAT containing larger adipocytes as compared with VAT [4]. Until recently the ideas presenting relationship of regional adiposity with insulin senisitivity/resistance were not consistent and studies differed in the assessment of the importance of WAT and BAT. Some studies have suggested that the majority of metabolic activity in the adipose tissue belongs to WAT which is the most critical determinant of insulin sensitivity [5], whereas others have indicated the dominant role of BAT $[6,7]$.

The lack of consistent findings is probably attributed to the use of different methodologies (eg. different type of liposuction procedure), small number of subjects, not homogenous study groups, differences in patient's lifestyle (exercise, diet), difficulties in maintaining stable body mass, and low sensitivity of an examination. At the moment, most authors agree that there is a positive correlation between accumulation of VAT and development of insulin resistance, while SAT determines leptin secretion which indirectly reflects the level of insulin sensitivity in the body [8].

\section{Liposuction procedure, metabolic change and maintenance of body weight after surgery}

Classic liposuction also known as suction-assisted liposuction (SAL) uses aspiration techniques to break down and draw the fat cells out of the body. In this method small cannula is inserted through a small incision and attached to a vacuum device. There are many different types of liposuction according to the volume of infiltration or wetting solution injected before the surgery: dry, wet, superwet and tumescent technique. Moreover, the surgery could be modified by the new technologies such as power assisted liposuction (PAL), ultrasound assisted liposuction (UAL) and laser assisted liposuction $(\mathrm{LAL})$. According to the volume of solution aspirated liposuction could be divided into two categories: large volume liposuction ( $>5$ liters aspirated) and small volume liposuction ( $<5$ liters aspirated). The most popular liposuction types are tumescent and superwet techniques with minimal risk of bleeding complications and small volume fluid infusions during surgery. Tumescent liposuction is performed under local anesthesia after subcutaneous infusion of fluid (most commonly containing saline, lidocaine, and epinephrine, with or without sodium bicarbonate) in a ratio of $2-3 \mathrm{~mL}$ of infiltrate to $1 \mathrm{ml}$ of aspirate; the endpoint of infiltration is tissue turgor [9]. For the best results liposuction candidates should be healthy and physically fit and not more than 20 pounds overweight, their skin should be firm and elastic. The major contraindications include and are not limited to: severe cardiovascular disease, severe coagulation disorders, pregnancy or less than 6 months post-partum, eating disorders, psychiatric problems, morbid obesity, serious life stress within last 6 months [10]. The indications and contraindications for liposuction summarizes Table 1.

Liposuction surgically removes only SAT and has almost no effect on VAT depots. Up to $50 \%$ of SAT is localized in the deep layer and, during abdominal liposuction, this is the predominant removed tissue. Some 
Table 1. General indications and contraindications for liposuction

\begin{tabular}{|c|c|c|}
\hline Indications & Absolute contraindications & Relative contraindications \\
\hline Aesthetic indications; reduced body mass & Psychiatric diseases; eating disorders & Previous liposuction complications \\
\hline Multiple systemic lipomatosis & Morbid obesity & $\begin{array}{l}\text { Some medications which cannot } \\
\text { or will not be stopped }\end{array}$ \\
\hline Dercum's disease & Unrealistic expectations & Previous liposuction in areas "of interest" \\
\hline Lipomas & Ucontrolled drug or alcohol addiction & Lifestyles which cannot or will not be changed \\
\hline Chronic Lymphedema & $\begin{array}{l}\text { Use of some specific medications, which cannot be } \\
\text { stopped }\end{array}$ & $\begin{array}{l}\text { More than } 50 \text { pounds weight } \\
\text { loss during last year }\end{array}$ \\
\hline Axillary hyperhidrosis & Pregnancy or less than 6 months post-partum period & Excessive smoking or alcohol use \\
\hline Post-ablative surgery & Immunosuppressive therapy (steroids) & Some psychiatric and/or social problems \\
\hline \multirow[t]{6}{*}{ Gynecomasty } & Anticoagulant therapy & \\
\hline & $\begin{array}{l}\text { Major surgery and/or general anesthesia within last } \\
\qquad 6 \text { months }\end{array}$ & \\
\hline & Stroke or heart attack within last 6 months & \\
\hline & Allergy to lidocaine & \\
\hline & Serious life stress within last 6 months & \\
\hline & Uncontrolled diabetes & \\
\hline
\end{tabular}

authors suggest that deep layers of SAT are functionally similar to VAT and the amount of deep subcutaneous abdominal adipose tissue is strongly related to insulin resistance in a manner nearly identical to that of visceral adiposity [11, 12]. The assessment whether liposuction is successful in a longitudinal weight management is the first step toward potential metabolic benefit analysis. In 2001 Commons et al. reviewed 631 cases of liposuction that was performed in the same hospital by the same surgeon over 12 years, with an average follow up 1 year. More than $80 \%$ of patients maintained stable body weight within 12 months from surgical procedure [13]. Contrary to this report, there are studies describing rebuilding of removed fat and its redistribution to the abdominal and visceral regions with negative impact on metabolic parameters and risk factors for coronary artery disease. In prospective analysis of fat distribution Hernandez et al. observed an initial decrease in abdominal SAT (6 weeks after liposuction) with subsequent significant increase in SAT and WAT after 1 year from surgery. Tested metabolic variables (serum adiponectin, free fatty acids, glucose, insulin, triglycerides, sensitivity to insulin) were not significantly changed except an initial drop in leptin level, which after 12 months returned to its basic concentrations. This study, however, was conducted on small study group $(n=14)$ and the type of surgery was small-volume liposuction (less than 5L of aspirate) [14].

It has been documented that large volume liposuction enhances insulin sensitivity markedly, lowers insulinemia and additionally reduces circulating markers of vascular inflammation [15]. The beneficial effect on insulin sensitivity persists over months from surgery $[16,17]$.

The number of study participants is one of the major determinants for statistical power and sensitivity of analysis. In a large clinical study (123 obese women) D'Andrea et al. observed positive metabolic changes after 21 and 90 days from large volume liposuction. They noted significantly improved insulin sensitivity, resting metabolic rate, serum adipocytokines, and level of inflammatory markers. The changes were correlated with the decrease in fat mass and waist-hip ratio and remained relatively stable [18]. Rizzo et al. evaluated the effects of dermolipectomy after 40 days from the surgery. They found significant decline in plasma resistin, and inflammatory markers (IL6, IL10, TNF) and an increase in plasma adiponectin. Moreover, observed metabolic changes were accompanied by an improvement in insulin-mediated glucose uptake, substrate oxidation and degree of inflammation [19].

Contrary to these findings, a number of studies reported no, or even diverse effects of liposuction on metabolic profile. They underlined that only negative energy balance induced by diet and/or exercise, not simply a decrease in the mass of adipose tissue, is critical for achieving the metabolic benefits of weight loss. Weight loss in response to energy deficit decreases VAT mass, production of proinflammatory cytokines, size of adipocytes, helps to reduce intrahepatic and myocellular fat, and undoubtedly, these changes bring significant improvement in most of the metabolic parameters [20]. In addition, some authors report post-liposuction adipose tissue regain and redistribution, preferentially in the abdominal regions of VAT, after months from the 
Table 2. General metabolic activities of adiponectin, leptin and resistin in humans

\begin{tabular}{|c|c|c|}
\hline Hormone & Site of production & Metabolic activity \\
\hline Adiponectin & white adipose tissue & $\begin{array}{l}\text { - increases fatty acids oxidation } \\
\text { - inhibits hepatic gluconeogenesis } \\
\text { - increases insulin sensitivity and glucose uptake } \\
\text { - has anti-inflammatory and anti-atherogenic activity }\end{array}$ \\
\hline Leptin & $\begin{array}{l}\text { white adipose tissue, brown adipose tissue, } \\
\text { stomach, placenta, skeletal muscle }\end{array}$ & $\begin{array}{l}\text { - inhibits appetite and enhances thermogenesis } \\
\text { - stimulates sympathetic activity increasing rate of metabolism } \\
\text { - inhibits insulin secretion } \\
\text { - increases insulin sensitivity } \\
\text { - increases lipolysis } \\
\text { - decreases hepatocyte lipogenesis preventing from lipotoxicity }\end{array}$ \\
\hline Resistin & $\begin{array}{l}\text { mononuclear blood cells, white adipose tissue, } \\
\text { skeletal muscle, pancreas }\end{array}$ & $\begin{array}{l}\text { - pro-inflammatory molecule } \\
\text { - mediates insulin resistance } \\
\text { - other metabolic functions are still unclear }\end{array}$ \\
\hline
\end{tabular}

surgery. This, in turn, may possibly result in consequences of metabolic dysregulation after surgery [14, 21].

For better understanding of the metabolic alterations after liposuction a number of studies determined its effect on major adipokines: adiponectin, leptin and resistin. These adipose tissue derived hormones are involved in maintenance of metabolic homeostasis (Table 2).

\section{Adiponectin}

Adiponectin is a hormone/cytokine primarily produced in the WAT. Changes in its serum concentration are associated with metabolic profile and risk factors for cardiovascular disorders. Serum level of adiponectin is reversibly correlated to BMI, insulinemia and triglycerides. The number of studies have shown that adiponectin increases insulin sensitivity via enhancement of fatty acids oxidation and inhibition of hepatic gluconeogenesis, and exhibits anti-inflammatory and antiatherogenic activity in blood vessels $[22,23]$. There are three forms of this hormone: LMW (low molecular weight), MMW (middle molecular weight), and HMW (high molecular weight). It has been shown that antidiabetic and antiatherogenic properties of adiponectin are related to HMW activity [24, 25].

A number of authors demonstrated that surgical removal of fat improves metabolic parameters like insulin sensitivity, and increases adiponectin secretion [18, 26]. As reported by Giugliano et al., super wet technique of liposuction was associated with improvement in insulin sensitivity measured by HOMA-IR (Homeostasis Model Assessment - Insulin Resistance), reduction in circulating markers of vascular inflammation (IL6, IL18, TNF alfa, CRP), and elevated serum adiponectin within six months of stable body weight after liposuction. Moreover, there was a positive correlation between the amount of fat aspirate and adiponectin and changes in HOMA [27]. Similar findings were described by Maher et al. after 12 weeks of observation. Using tumescent technique and aspirating more than $5 \mathrm{~L}$ of fat they noted that insulin sensitivity has greatly improved in obese women with and without coexisting diabetes. The level of adiponectin was increased in both groups, but not significantly in patients without diabetes [28]. It has been shown that weight reduction increases adiponectin plasma level. Thus, after liposuction an inhibitory effect of obesity on its production is possibly "turned off" [26]. On the other hand, there are negative studies that deny the existence of metabolic benefits and elevated adiponectin levels in response to liposuction. They underline that regulation of adipokine production is multifactorial in response to negative energetic balance that could be induced by exercise and/or dietary restriction only. Physical effort as a nonpharmacological and nonsurgical intervention evidently reduces VAT level, even if body mass has not been decreased so markedly $[20,29]$. Decreased size of adipocytes decreases leptin release, which is a well known factor inhibiting adiponectin production, the other mechanisms involve improvement in BAT blood flow, antioxidant effects, and high catecholamine level [30]. Most recent data indicate that irrespectively of exercise training small-volume liposuction down regulates the expression of adiponectin genes in SAT and its serum level [31]. To clarify these discrepancies, however, further studies performed on large population cohorts and including mechanistic insights are required.

\section{Leptin}

Leptin is a hormone secreted primarily by WAT. It acts as a satiety factor in signaling whole body energy balance. High levels of circulating leptin signal adequate 
energy stores whereas low levels are consistent with an energy deficit. At the hypothalamic level leptin inhibits appetite and enhances thermogenesis by decreasing the activity of orexigenic neurons (Npy/AgRP/ GABA containing neurons) and increasing the activity of the anorexigenic neurons (POMC/CART containing neurons). Leptin, through its central activity, favors catabolism in BAT which is partially a consequence of its stimulatory effect on sympathetic nervous system [32]. Barzilai et al. showed that peripheral leptin stimulates its receptors in VAT and selectively decreases visceral adiposity preventing from development of an insulin resistance [33]. The current data clearly indicate counterregulation of insulin by leptin through inhibition of insulin secretion, increase in hepatic insulin extraction, suppression of insulin lipogenesis by leptin lipolysis, and modulation of peripheral tissue and brain sensitivity to insulin action [34, 35]. Decreasing hepatocyte lipogenesis leptin prevents development of lipotoxicity, the condition which often contributes to the insulin resistance. Leptin-mediated inhibitory feedback on insulin secretion is related with decrease in adipogenesis and parallel increase in overall insulin sensitivity. The direct stimulatory effect of leptin on glucose uptake and increased insulin sensitivity is limited to muscle, because prolonged exposure of adipocytes to leptin results in a loss of insulin sensitivity and an inhibition of insulin stimulated lipogenesis. $[36,37]$. The reciprocal effects on synthesis of leptin and insulin are regulated by complex mechanisms that occur within adiopoinsular axis. Both insulin and glucose increase the synthesis of leptin, while leptin alone acts as a potent inhibitor for insulin secretion preventing occurrence of hyperinsulinemia and lipotoxicity. On the other hand, hyperinsulinemia interferes with leptin signaling at the peripheral receptor level and facilitates leptin resistance [38].

Normally leptin concentrations tend to increase with increasing adiposity, however, in obese individuals this interaction is somehow inhibited and leptin resistance develops [39]. As suggested by authors, this resistance abolishes only selected actions of leptin. Enriori et al. demonstrated that although hyperleptinemia in obese subjects was linked with resistance to anorexigenic effects of leptin, the ability to activate dorsomedial nucleus of hypothalamus and sympathetic drive to BAT was sustained [40]. Additionally, peripheral resistance to leptin in WAT was also widely described [41]. Peripheral leptin resistance may contribute to decreased leptin ability to modify the adipocyte insulin responsiveness and to maintain normal basal rate of lipolysis.
Almost all cases of obesity contribute to increased leptin resistance and deregulation of its central anorexigenic activities. Thus, it has been postulated that liposuction by decreasing adiposity could possibly improve both leptin and insulin sensitivity. Most authors agree that one of the early effects of liposuction is lowered leptin level but the relationship between hypoleptinemia and insulin sensitivity is not so evident. In animal model, Schrebier et al. reported an increasing trend of leptin level at 42 day after liposuction [42]. In humans, SAL liposuction was followed by an early drop in serum leptin concentration (after 1 week), which additionally correlated with voluntary changes in energy intake. The effect was not significant after 6 weeks from surgery [43]. Another study demonstrated the effect of low level laser therapy (LLLT) on serum leptin and lipid profile in overweight and obese women. Authors observed increase in serum triglycerides and decrease in leptin level. However, the effect was limited to overweight patients only (BMI 25-29.9) [44]. In an open parallel-group clinical trial Robles-Cervantes et al. tested metabolic profile in two groups of obese women: "liposuction plus diet" and "diet only". They found a marked decrease in leptin level 1 month after liposuction, however this change was not correlated with insulin sensitivity Described by many authors delayed return of leptin concentrations to the levels before surgery with simultaneous maintenance of body weight (when yo-yo effect is absent) may indicate potential metabolic benefits resulting from improved peripheral and central leptin sensitivity [36]. In meta-analysis of 15 studies on cardiovascular metabolic markers after suction assisted lipectomy only leptin and fasting insulin were the variables that were significantly associated with the amount of aspirated fat, whereas the other factors such as inflammatory markers were not [45].

\section{Resistin}

Resistin is yet another cytokine that may contribute to obesity and impaired metabolic profile. It was originally named for its resistance to insulin, and was proposed to impair glucose tolerance and insulin action, but the exact role of this peptide in humans still remains unclear. In animal model, resistin suppresses insulin stimulated glucose uptake and induces insulin resistance [46]. Excessive adiposity increases expression of resistin genes in adipose tissue and resistin works as a feedback regulator of adipogenesis in rats [47]. However, unlike the rodents, human resistin is predominantly expressed in human mononuclear cells in response to 
inflammatory stimuli and, because of that, cannot be considered as a fat-derived cytokine [48]. On the other hand, in vitro studies on isolated human adipocytes have shown that antigenic stimuli increase secretion of resistin from adipose tissue. Moreover, culturing adipocytes with recombinant human resistin evokes proinflammatory cytokine release and up-regulates mediators of insulin signaling pathway [49]. Most studies on genetic programming confirm the correlation between increased gene expression, inflammatory response and risk for diabetes [50]. In 2001 Stepan et al. described the link between diabetes and level of serum resistin. They have found that administration of anti-resistin antibody improves blood sugar and insulin action in mice with diet-induced obesity. Moreover, treatment of normal mice with recombinant resistin impaired glucose tolerance and insulin action [51]. According to recent large case-controlled clinical studies on humans, elevated levels of resistin are associated with the development of diabetes type 2, possibly through inflammatory processes, but the mechanistic insights require further evaluation [52]. Resistin levels appear to correlate positively with SAT and majority of studies confirm this relationship [53]. A longitudinal analysis of patients on a weight reduction program including dieting and exercise, has brought an evidence that serum resistin change is positively correlated with changes in BMI, body fat, fat mass, visceral fat area, and mean glucose and insulin in patients [54]. Little is known about the effect of abdominal lipoplasty on resistin level. D'Andrea et al. reported decrease in serum resistin after large volume liposuction. Their report was based on observation of a large study group, so they were able to detect even discrete effect of liposuction on levels of studied variables [18]. Ma et al. confirmed beneficial metabolic effects of liposuction describing lowered resistin level with no change in inflammatory markers (CRP and IL6), and improved insulin sensitivity after 3 months from surgery. However, their study group was very small and consisted of 16 individuals only [55]. Similar effect but after dermolipectomy was presented by Rizzo et al. who found that after 40 days following surgery decrease in serum resistin was accompanied by a significant improvement in insulinmediated glucose uptake [19]. Ramos-Gallardo et al. suggested that in patients with impaired lipid profile, liposuction can reduce the metabolism of cholesterol by lowering the level of resistin, which is known to increase the production of LDL and degrades LDL receptors in the liver [56].

\section{Summary}

Numerous clinical and experimental studies have brought conflicting findings about abdominal liposuction surgery and its metabolic effects including adipokines secretion and insulin sensitivity. The possible explanation includes differences in a lifestyle of examined subjects (diet, physical activity), difficulties in maintaining stable body mass, experimental methodology (eg. differences in the follow-up time after surgery or the methods used to test for insulin sensitivity), and the type of liposuction procedure used (eg. large vs. small volume liposuction). Moreover, most of published observations was based on examination of the small number of study participants. Thus, the current data cannot bring a clear evidence suggesting that liposuction itself results in important metabolic outcomes, on the other hand, the possibility that liposuction may serve as a new strategy for rapid restoration of impaired metabolic profile cannot be completely excluded. Some of the scientists suggest its additive/facilitating effect when combined with reduced calorie intake and increased energy expenditure [18]. Indeed, more trials performed on large population, that address the long-term effects of SAL on metabolic markers and are similar in experimental design and study homogeneity, are required to evaluate this concept in details.

\section{Acknowledgements}

\section{Conflict of interest statement}

The authors declare that there is no conflict of interest in the authorship or publication of contribution.

\section{Funding sources}

There are no sources of funding to declare.

\section{References}

1. Kershaw EE, Flier JS. Adipose Tissue as an Endocrine Organ. J Clin Endocrinol \& Metab. 2004;89(6):25482556.

2. Siemińska L. Adipose tissue. Pathophysiology, distribution, sex differences and the role in inflammation and cancerogenesis. Pol J Endocrinol. 2007;58(4):330-342.

3. Alberti KG, Zimmet PZ. Definition, diagnosis and classification of diabetes mellitus and its complications. Part 1: diagnosis and classification of diabetes mellitus provisional report of a WHO consultation. Diabet Med. 1998;15:539-553.

4. Wajchenberg BL. Subcutaneous and visceral adipose tissue: their relation to the metabolic syndrome. Endocr Rev. 2000;21(6):697-738.

5. Abate N, Garg A, Peshock RM, Stray-Gundersen J, Grundy SM. Relationships of generalized and regional adiposi- 
ty to insulin sensitivity in men. J Clin Invest. 1995;96:8898.

6. Park KS, Rhee BD, Lee KU, Kim SY, Lee HK, Koh CS, Min HK. Intraabdominal fat is associated with decreased insulin sensitivity in healthy young men. Metabolism. 1991;40:600-603.

7. Rendell M, Hulthen UL, Tornquist C, Groop L, Mattiasson I. Relationship between abdominal fat compartments and glucose and lipid metabolism in early postmenopausal women. J Clin Endocrinol Metab. 2001;86:744 -749.

8. Cnop M, Landchild MJ, Vidal J, Havel PJ, Knowles NG, Carr DR, Wang F, Hull RL, Bovko EJ, Retzlaff BM, Walden $\mathrm{CE}$, Knopp RH, Kahn SE. The concurrent accumulation of intra-abdominal and subcutaneous fat explains the association between insulin resistance and plasma leptin concentrations: distinct metabolic effects of two fat compartments. Diabetes. 2002;51:1005-1015.

9. Rohrich RJ, Kenkel JM, Janis JE, Beran SJ, Fodor PB. An update on the role of subcutaneous infiltration in suction assisted lipoplasty. Plast Reconstr Surg. 2003;111:926-7.

10. American Society for Aesthetic Plastic Surgery. ASAPS Statistics on cosmetic surgery. New York: American Society for Aesthetic Plastic Surgery, 2003.

11. Kelley DE, Thaete FL, Troost F, Huwe T, Goodpaster BH. Subdivisions of subcutaneous abdominal adipose tissue and insulin resistance. Am J Physiol Endocrinol Metab. 2000;278:E941-E948.

12. Perez RA. Liposuction and diabetes type 2 development risk reduction in the obese patient. Med Hypotheses. 2007;68(2):393-396.

13. Commons GW, Halperin B, Chang CC. Large-volume liposuction: a review of 631 consecutive cases over 12 years. Plast Reconstr Surg. 2001;108:1753-1763.

14. Hernandez TL, Kittelson JM, Law CK, Ketch LL, Stob NR, Lindstrom RC, Scherzinger A, Stamm ER, Eckel RH. Fat Redistribution Following Suction Lipectomy: Defense of Body FAT and Patterns of Restoration. Obesity. 2011;19:1388-1395.

15. Giugliano G, Nicoletti G, Grella E, Giugliano F, Esposito $K$, Scuderi N, D’Andrea F Effect of liposuction on insulin resistance and vascular inflammatory markers in obese women. Br J Plast Surg. 2004;57(3):190-194.

16. Giese SY, Bulan EJ, Commons GW, Spear SL, Yanovski JA. Improvements in cardiovascular risk profile with large -volume liposuction: a pilot study. Plast Reconstr Surg. 2001;108:510-520.

17. González-Ortiz M, Robles-Cervantes JA, Cárdenas-Camarena $L$, Bustos-Saldańa R, Martínez-Abundis E. The effects of surgically removing subcutaneous fat on the metabolic profile and insulin sensitivity in obese women after large-volume liposuction treatment. Horm Metab Res. 2002;34:446-449.

18. D'Andrea F, Grella R, Pizzo MR, Grella E, Nicoletti G, Barbieri M, Paolisso G. Changing the metabolic profile by large-volume liposuction: a clinical study conducted with 123 obese women. Aest Plast Surg. 2005;29(6):472-8; discussion 479-80, 481.

19. Rizzo MR, Paolisso G, Grella R, Barbieri M, Grella E, Ragno E, Grella R, Nicoletti G, D'Andrea F. Is dermolipectomy effective in improving insulin action and lowering inflammatory markers in obese women? Clin Endocrinol. 2005;63(3):253-258.
20. Klein S, Fontana L, Young VL, Coggan AR, Kilo C, Patterson PW, Mohammed BS. Absence of an effect of liposuction on insulin action and risk factors for coronary heart disease. N Engl J Med. 2004;350(25):2549-57.

21. Weber RV, Buckley MC, Fried SK, Kral JG. Subcutaneous lipectomy causes a metabolic syndrome in hamsters. Am J Physiol Regul Integr Comp Physiol. 2000; 279: R936R943.

22. Diez JJ, Iglesias P. The role of the novel adipocyte-derived hormone adiponectin in human disease. Eur J Endocrinol. 2003;148:293-300.

23. Lihn AS, Pedersen SB, Richelsen B. Adiponectin: action, regulation and association to insulin sensitivity. Obes Rev. 2005;6(1):13-21.

24. Moroi M, Akter $S$, Nakazato R, Kunigasa T, Masai H, Furuhashi T, Fukusa H, Koda E, Sugi K, Jesmin S. Lower ratio of high-molecular-weight adiponectin level to total may be associated with coronary high-risk plaque. BMC Research Notes. 2013;6:83. http://www.biomedcentral. com/1756-0500/6/83.

25. Heidemann C, Sun Q, van Dam RM, Meigs JB, Hang C, Tworoger SS, Mantzoros CS, Hu FB. Total and high-molecular-weight adiponectin and resistin in relation to the risk for type 2 diabetes in women. Ann Intern Med. 2008;149(5):307-16.

26. Yang WS, Lee WJ, Funahashi T, Tanaka S, Matsuzawa Y, Chao CL, Chi-Ling Chen CL, Tai TY, Lee-Ming Chuang LM. Weight reduction increases plasma levels of an adipose-derived anti-inflammatory protein, Adiponectin. J Clin Endocrinol Metab. 2001;86(8):3815- 3819 (Erratum. 2002, J. Clin Endocrinol Metab. 2001;87:1626.

27. Giugliano G, Nicoletti G, Grella E, Giugliano F, Esposito $K$, Scuderi N, D`Andrea F. Effect of liposuction on insulin resistance and vascular inflammatory markers in obese women. Br J Plast Surg. 2004;57(3):190-194.

28. Maher A, Kamel I. Effect of large-volume abdominal liposuction on serum adiponectin level and its metabolic consequences in obese women. Egypt J Plast Reconstr Surg. 2009;33(2):201-208.

29. Mohammed BS, Cohen S, Reeds D, Young VL, Klein S. Long-term effects of large-volume liposuction on metabolic risk factors for coronary heart disease. Obesity. 2008;16(12):2648-2651.

30. Berggren JR, Hulver MW, Houmard JA. Fat as an endocrine organ: influence of exercise. J App Physiol. 2005;99(2):757-764.

31. Solis MY, Artioli GG, Montag E, Painelli V, Saito FL, Lima FR, Roschel H, Gualano B, Lancha AH, Benatti FB. The liposuction-induced effects on adiponectin and selected cytokines are not affected by exercise training in women. Int J Endocrinol. 2014;2014:315382.

32. Correira MLG, Morgan DA, Mitchell JL, Sivitz WI, Mark AL., Haynes WG. Role of corticotrophin-releasing factor in effects of leptin on sympathetic nerve activity and arterial pressure. Hypertension. 2001;38:384-388.

33. Barzilai N, Wang J, Massilon D, Vuguin P, Hawkins M, Rossetti L. Leptin selectively decreases visceral adiposity and enhances insulin action. J Clin Invest. 1997;100:31053110

34. Borer KT. Counterregulation of insulin by leptin as key component of autonomic regulation of body weight. World J Diabetes. 2014;5:606-629. 
35. Harris RB. Direct and indirect effects of leptin on adipocyte metabolism. Biochim Biophys Acta. 2014;1842(3):41423.

36. Muller G, Ertl J, Gerl M, Preibisch G. Leptin impairs metabolic actions of insulin in isolated rat adipocytes. J Biol Chem. 1997; 272:10585-10593.

37. Yildiz BO, Haznedaroglu IC. Rethinking leptin and insulin action: therapeutic opportunities for diabetes. Int J Biochem Cell Biol. 2006; 38(5-6):820-830.

38. Kellerer M, Lammers R, Fritsche A, Strack V, Machicao F, Borboni P, Ullrich A, Haring HU. Insulin inhibits leptin receptor signalling in HEK293 cells at the level of janus kinase-2: a potential mechanism for hyperinsulinaemiaassociated leptin resistance. Diabetologia. 2001;44:11251132.

39. Jequier E. Leptin signaling, adiposity, and energy balance. Ann N Y Acad Sci. 2002;967:379-88.

40. Enriori PJ, Sinnayah P, Simonds SE, Garcia Rudaz C, Cowley MA. Leptin action in the dorsomedial hypothalamus increases sympathetic tone to brown adipose tissue in spite of systemic leptin resistance. J Neurosci. 2011;31:12189-12197.

41. Wang Z, Zhou YT, Kakuma T, Lee Y, Kalra SP, Kalra PS, Pan W, Unger RH. Leptin resistance of adipocytes in obesity: role of suppressors of cytokine signaling. Biochem Biophys Res Commun. 2000;277:20-26.

42. Schreiber JE, Singh NK, Shermak MA. The effect of liposuction and diet on ghrelin, adiponectin, and leptin levels in obese Zucker rats. Plast Reconstr Surg. 2006;117(6):1829-1835.

43. Talisman R, Belinson N, Modan-Moses D, Canti H, Orenstein A, Barzilai Z, Parret G. The Effect of Reduction of the Peripheral Fat Content by Liposuction-Assisted Lipectomy (SAL) on Serum Leptin Levels in the Postoperative Period: A Prospective Study. Aest Plast Surg. 2001;25(4):262-265.

44. Salem ES, Serry ZM, Tawfik MS, HF Aboel Magd, Youssef SS. The photo biological effect of low level laser therapy on serum level of leptin, cholesterol and triglycerides in overweight and obese females. Arab J Nuc Sci Apel. 2013;46(3):307-312.

45. Danilla S, Longton C, Valenzuela K, Cavada G, Norambuena $\mathrm{H}$, Tabilo C, Erazo C, Benitez S, Sepulveda S, Schulz $R$, Andrades P. Suction-assisted lipectomy fails to improve cardiovascular metabolic markers of disease: A meta -analysis. Journal of Plastic, Reconstructive \& Aesthetic Surgery. 2013;66:1557-1563.

46. Shuldiner AR, Yang R, Gong DW. Resistin, obesity, and insulin resistance-the emerging role of the adipocyte as an endocrine organ. N Engl J Med. 2001;345:13451346.

47. Li J, Yu X, Pan W, Unger RH. Gene expression profile of rat adipose tissue at the onset of high-fat-diet obesity. Am J Phys Endocrinol Metab. 2002;282:E1334-E1341.

48. Patel L, Buckels AC, Kinghorn IJ, Murdock PR, Holbrook JD, Plumpton C, Macphee $\mathrm{CH}$, Smith SA. Resistin is expressed in human macrophages and directly regulated by PPAR gamma activators. Biochem Biophys Res Commun. 2003;300:472-476.
49. Kusminski CM, da Silva NF, Creely SJ, Fisher FM, Harte AL, Baker AR, Kumar S, McTernan PG. The in vitro effects of resistin on the innate immune signaling pathway in isolated human subcutaneous adipocytes. J Clin Endocrinol Metab. 2007;92:270-276.

50. Osawa H, Yamada K, Onuma H, Murakami A, Ochi M, Kawata $H$, Nishimiya $T$, Niiya $T$, Shimizu I, Nishida W, Hashiramoto M, Kanatsuka A, Fujii Y, Ohashi J, Makino $\mathrm{H}$. The $\mathrm{G} / \mathrm{G}$ genotype of a resistin single-nucleotide polymorphism at -420 increases type 2 diabetes mellitus susceptibility by inducing promoter activity through specific binding of Sp1/3. Am J Hum Genet. 2004;75:678686.

51. Steppan CM, Bailey ST, Bhat S, Brown EJ, Banerjee RR, Wright CM, Patel HR, Ahima RS, Lazar MA. The hormone resistin links obesity to diabetes. Nature. 2001;409:307312.

52. Chen BH, Song $Y$, Ding EL, Roberts CK, Manson JE, Rifai N,. Buring JE, Gaziano JM, Liu S. Circulating levels of resistin and risk of type 2 diabetes in men and women: results from two prospective cohorts. Diabetes. 2009;32:329-334.

53. Won JC, Park CY, Lee WY, Lee ES, Oh SW, Park SW. Association of plasma levels of resistin with subcutaneous fat mass and markers of inflammation but not with metabolic determinants or insulin resistance. J Korean Med Sci. 2009;24:695-700.

54. Azuma K Katsukawa F, Oguchi S, Murata M, Yamazaki H, Shimada A, Saruta T. Correlation between serum resistin level and adiposity in obese individuals. Obes Res. 2003;11:997-1001.

55. Ma GE, Liu P, Lei H, Chen J, Liu ZJ. Effect of liposuction on adipokines, inflammatory markers and insulin resistance. Zhonghua Zheng Xing Wai Ke Za Zhi. 2010;26(1):26-8.

56. Ramos-Gallardo G, Pérez Verdin A, Fuentes M, Godinez Gutierrez S, Ambriz-Plascencia AR, Gonzalez-Garcia I, Gomez-Fonseca SM, Madrigal R, Gonzalez-Reynoso LI, Figueroa S, Toscano Igaruta X, Jimenez Gutierrez DF. Effect of abdominoplasty in the lipid profile of patients with dyslipidemia. Plast Surg Int. 2013;2013:861348.

Acceptance for editing: 2015-11-10 Acceptance for publication: 2015-12-31

Correspondence address: Magdalena Gibas-Dorna Department of Physiology Poznan University of Medical Sciences 5 Swiecickiego Str., 60-781 Poznan, Poland phone: +48618546540 email: physioplus@wp.pl 